\title{
Nuevo orden internacional a inicios de la segunda década del siglo XXI
}

\author{
New international order at the beginning \\ of the second decade of the $21^{\text {st }}$ Century
}

Julio Ramírez Montañez y Jaime Sarmiento Suárez ${ }^{* *}$

\begin{abstract}
RESUMEN:
El objetivo del presente artículo de reflexión fue analizar el nuevo contexto internacional y el nuevo escenario con el que se enfrenta la comunidad internacional en la víspera del inicio de la segunda década del siglo xxi. El Orden Internacional ha sufrido una transformación en los últimos años, generada por una crisis del multilateralismo heredado del período de la post guerra. Esta crisis del multilateralismo se ha visto materializada mediante un proceso de desglobalización de la economía mundial, fundamentada principalmente en políticas neoproteccionistas que han conducido a una desregionalización. Esta es una reflexión sobre la situación geopolítica generada en los últimos años, donde el mundo avanza progresivamente hacia nuevos escenarios de intercomunicación en el orden mundial, hacia diferentes horizontes geopolíticos, en un contexto de redistribución de supremacías y transformaciones en los paradigmas económicos. Estos cambios están sustentados en un proceso de desglobalización de la economía mundial, del resurgimiento del proteccionismo y de la desregionalización del comercio internacional mediante la renegociación de acuerdos comerciales.
\end{abstract}

* Coordinador de Investigación Escuela de Economía, Administración y Negocios. Universidad Pontificia Bolivariana, Bucaramanga. Magister en Relaciones Internacionales, Flinders University of South Australia. ORCID: 0000-0003-0116-3330.Correo electrónico: julio.ramirez@upb. edu.co.

** Docente investigador de la Universidad Pontificia Bolivariana - Seccional Bucaramanga. Doctor (c) en Gestión de la Tecnología y la Innovación de la Universidad Pontificia Bolivariana. Magister en Administración de la Universidad Autónoma de Bucaramanga - Instituto Tecnológico de Monterrey. Ingeniero de Sistemas e Ingeniero Industrial de la Universidad Industrial de Santander. ORCID: 0000-0003-0313-5675. Correo electrónico: jaime.sar@upb.edu.co. Recibido: 20 de septiembre de 2019. Modificado: 08 de junio de 2020. Aceptado: 29 de junio de 2020. 
Palabras Claves: Nuevo Orden Internacional - Desglobalización

- Desregionalización - Neo-proteccionismo

\section{ABSTRACT:}

The purpose of this reflection article was to analyze the new international context and the new scenario with which the international community is facing on the eve of the beginning of the second decade of the 21st century. The International Order has undergone a transformation in recent years generated by a crisis of multilateralism inherited from the post-war period. This crisis of multilateralism has been materialized through a process of de-globalization of the world economy based mainly on neo-protectionist policies that have led to de-regionalization. This is a reflection on the geopolitical situation generated in recent years, where the world is progressively moving towards new scenarios of intercommunication in the world order, towards different geopolitical horizons, in a context of redistribution of supremacies and transformations in economic paradigms. These changes are underpinned by a process of de-globalization of the world economy, the resurgence of protectionism and the de-regionalization of international trade through the renegotiation of trade agreements.

Keywords: New International Order - Des globalization - Des regionalization - Neo-protectionism. 


\section{Nuevo Orden InTERnacional}

En la década que termina han acontecido sucesos que han afectado el contexto político y económico internacional. La nueva política exterior de algunas potencias globales, como Estados Unidos y el Reino Unido, han puesto en tela de juicio las doctrinas basadas en el librecambismo y la apertura de mercados, las cuales habían sido promulgadas y en algunos casos impuestas por estos dos países durante los siglos xIx y xx.

Las implicaciones en la economía global de sucesos como el llamado BREXIT, donde los ciudadanos del Reino Unido, mediante voto popular, decidieron retirarse del bloque económico y político de la Unión Europea, así como el nuevo direccionamiento de la política comercial de Estados Unidos durante la Administración Trump, caracterizada por la renegociación de los tratados de libre comercio y la aplicación de medidas proteccionistas encaminadas a salvaguardar la industria nacional, son solo dos ejemplos que reflejan la conformación de un nuevo panorama internacional.

En esta transición de los últimos años se ha producido un creciente rechazo al libre comercio en los países avanzados. El voto a favor del BRExIT en el Reino Unido, la victoria de Trump o el auge de los partidos No Tradicionales en Europa, refleja un sentimiento de rechazo a la apertura al comercio internacional, la inversión y la inmigración de amplias capas de la sociedad, que buscan recuperar la soberanía económica y comercial perdida levantando nuevas fronteras.

En este nuevo panorama internacional, en el plano comercial se da un resurgir del neo- proteccionismo que se traduce, cada vez más, en el rechazo por parte de la opinión pública a los nuevos acuerdos comerciales mega-regionales, sobre todo el Acuerdo Transpacífico de Cooperación Económica (TPP) y la Asociación Transatlántica para el Comercio y la Inversión y la Organización Mundial del Comercio (омC).

Para algunos autores, más allá que la llegada de esta nueva administración republicana a la Casa Blanca haya supuesto una convulsión para el sistema comercial mundial, lo cierto es que ya se estaban produciendo transformaciones importantes tanto en la geografía como en la gobernanza del comercio y las inversiones internacionales. En primer lugar, como explica Richard Baldwin en su libro "The Great Convergence", la naturaleza del comercio internacional ha sufrido una transformación radical en las últimas décadas. Este autor señala que la globalización se ha convertido en el chivo expiatorio de todos los males. A los tradicionales movimientos antiglobalización, que asocian el proceso al avance imparable del capitalismo, se han sumado más recientemente los populismos de todo corte - sobre todo los de ultraderecha-, que han encontrado 
en ella la bandera a la que amarrar su discurso nacionalista, proteccionista y xenófobo. (Baldwin, 2016).

Una de las víctimas más visibles de esta tendencia antiglobalización es el libre comercio. El libre comercio, después de décadas de dominio como doctrina global, ha venido perdiendo terreno en la esfera internacional y el proteccionismo ha venido ganando terreno, especialmente en la guerra comercial impulsada por Estados Unidos en contra de China.

El presente artículo está estructurado inicialmente en la primera sección para presentar las razones de la crisis del multilateralismo por la que atraviesa la comunidad internacional en la actualidad. En la segunda parte del artículo se presentan los rasgos más sobresalientes del proceso de desglobalización que viene afectando a la economía mundial. Posteriormente, se presenta la forma como se está implementando el neoproteccionismo por parte de los Estados más desarrollados, especialmente Estados Unidos y el Reino Unido. En la sección final del artículo se describe el proceso de desregionalización.

\section{i. Crisis del Multilateralismo}

El sistema internacional está viviendo una metamorfosis complicada, con altos niveles de incertidumbre y de transformaciones precipitadas, con cambios que implican reconfiguraciones geoeconómicas y geopolíticas a nivel global. (Serbin, 2018). El triunfo de Donald Trump en las elecciones estadounidenses; la victoria del referéndum del BREXIT en el Reino Unido; el ascenso de las fuerzas de ultraderecha en Francia, Holanda, Alemania y otros países europeos, así como el avance de organizaciones políticas nuevas de izquierda, como Unidos Podemos en España y Sryza en Grecia, expresan la creciente incapacidad de los gobiernos y partidos políticos social-demócratas y de derecha tradicionales para resolver las crisis y paliar los estragos de la globalización neoliberal en amplios sectores de su población (Guillen, 2018).

Sangiovanni \& Hofmann (2019) en su paper "Of the Contemporary Global Order, Crisis, and Change", publicado en el Journal of European Public Policy, argumentan que el orden global de la postguerra, basado en relaciones inter-estatales soberanas y en una economía global relativamente abierta, se encuentra en crisis y se está gestando una transformación y adaptación a un sistema más amplio y flexible de gobernanza global. Estos autores señalan que el orden mundial que se encuentra en crisis y nos rigió hasta la primera década del siglo xxi tuvo sus orígenes en el mundo de postguerra y se basó en tres principios fundamentales: soberanía nacional, el liberalismo económico y el multilateralismo. 
En cuanto a la soberanía nacional, los gobiernos nacionales han sido los principales agentes responsables de la elaboración y aplicación de normas y políticas internacionales (Barkin y Cronin 1994). Por su parte, el liberalismo económico ha promovido apertura económica y la interdependencia entre la mayoría de países de todo el mundo (Ikenberry 2018). Y para sostener estos principios, los gobiernos han respaldado una estrategia de multilateralismo, donde el orden se ha construido en torno a un conjunto de reglas y principios, acordados y consagrados por el derecho internacional, incluida la utilización de la fuerza militar (Guillen, 2018).

En ese mismo sentido, autores como Zelicovich comparten esa premisa argumentando que "el multilateralismo tal y como lo conocemos está en crisis" (Zelicovich, 2019. P. 90). Este diagnóstico es compartido por espectadores, estudiosos e investigadores de las relaciones internacionales, donde en la actualidad abundan las expresiones para referirse a este nuevo panorama geopolítico internacional.

El autor Jürgen Rüland utiliza el término de "multilateralismo disminuido" para referirse a una situación en la que, según él, el multilateralismo está caracterizado por "disputas cada vez más intensas acerca de la composición y la representación, las normas y los procesos de toma de decisiones", de manera que "las instituciones internacionales se han convertido cada vez más en arenas para las luchas de poder" (Rüland, 2012: 256).

En tanto, Edward Newman ha empezado a hablar de "malestar multilateral”, provocado por los retos a los que se enfrenta "el modelo convencional de soberanía estatal” (Newman, 2007: 15). De igual forma, Pascal Lamy, director general de la Organización Mundial del Comercio, ha advertido que "el multilateralismo está en una encrucijada", en el sentido de que "o avanza en el espíritu de los valores compartidos y la cooperación reforzada o nos enfrentaremos, a nuestro propio riesgo, a una retirada del multilateralismo".

Para García Moritán (2019), la erosión del multilateralismo ha fracturado equilibrios políticos y jurídicos del derecho internacional y se encuentra afectando la solución de cuestiones urgentes, como el cambio climático, los Objetivos del Milenio o la Agenda 2030 para el Desarrollo Sostenible, que se alargan en negociaciones interminables. En el campo de la seguridad internacional el panorama es igualmente desilusionante. Los acuerdos de desarme brillan por su ausencia.La carrera por armas nucleares y armas convencionales se ha acelerado en un espiral de graves consecuencias potenciales para la paz. También el espacio exterior se ha transformado en puja de poder militar.

Estas circunstancias, que destacan el predominio del unilateralismo o de un multilateralismo selectivo, ponen 
en evidencia la necesidad urgente de asumir el reto de liderar el objetivo de lograr un sistema multilateral global renovado, más representativo, sobre la base de reglas comunes y la recreación de instituciones creíbles, sólidas y eficaces (García Moritán, 2019).

La manera como se ha venido materializando este nuevo panorama internacional por parte del gobierno norteamericano es apostando menos a los macro-bloques comerciales, tipo Transatlantic Trade and Investment Partnership (TтIP) y Acuerdo Transpacífico de Cooperación Económica (тTP), y apostando a celebrar acuerdos bilaterales en los que tiene ventaja y más facilidad para imponer su posición. Con este tipo de acciones, el presidente Donald Trump ha querido propiciar la desintegración del multilateralismo $y$, de esta forma, ha socavado el propio liderazgo que su país ejerció desde la Segunda Guerra Mundial.

Otra muestra de la crisis del multilateralismo es la fragilidad en la que se encuentra la institución que ha servido de garante para el orden de libre comercio mundial: la Organización Mundial del Comercio. Dentro de la omc, durante años se ha tenido paralizado cualquier avance de la Ronda Doha. La amenaza principal cae ahora sobre el mecanismo de resolución de diferencias, específicamente sobre su Órgano de Apelación, cuya renovación está también paralizada. De no revertirse esta situación en diciembre de 2019 quedará tan solo uno de los siete árbitros de los que debe estar compuesto, con lo que el órgano dejaría de funcionar. La omc podría así perder el medio por el que busca garantizar un comercio basado en reglas compartidas y, por tanto, su credibilidad y eficacia. Buena parte del bloqueo procede, de nuevo, de Estados Unidos, no conforme con varias de sus resoluciones y siempre cuestionando los límites entre el multilateralismo y su propia soberanía.

Un punto sobresaliente que ha ayudado a esta crisis del multilateralismo es el fin de la hegemonía estadounidense. $\mathrm{Al}$ respecto, Dobat \& Leal (2019) manifiestan que el liderazgo de Estados Unidos se encuentra en un proceso de disminución en prácticamente todos los rubros, lo que lo imposibilita a seguir ejerciendo una hegemonía absoluta. Pero ni China ni algún otro país están en condiciones de ejercerla. Lo que diferencia a la nueva polarización del mundo de las anteriores formas de configuración del orden mundial, es que ahora no se basa en dos grandes bloques cerrados o semicerrados entre sí, sino dentro del espacio abierto de la globalización y la interdependencia comercial y financiera entre países. Ello hace que el balance de poder entre las dos grandes potencias dependa, en principio, del ritmo y la calidad del desarrollo interior, así como de las vinculaciones y alianzas exteriores, en la cual las relaciones de China con los países en desarrollo emergentes le otorgan ventaja. 


\section{Desglobalización}

Existen distintas posiciones que señalan a la globalización económica como la causa de las más relevantes discrepancias sociales a nivel global. Estas corrientes han venido siendo lideradas por movimientos sociales antiglobalización, los cuales vienen planteando la idea de la apertura de un período denominado "desglobalización internacional”, es decir, una etapa de repliegue en la interrelación e interdependencias de los Estados, prevaleciendo su plena soberanía sobre la exigencia normativa que plantean las organizaciones internacionales, los tratados internacionales de cooperación o el posicionamiento global de las compañías transnacionales que, según este enfoque, atentan contra la soberanía plena de los Estados (Copelli, 2018).

De acuerdo a González (2019), tras décadas de evolución desenfrenada de la globalización, la insatisfacción de un gran porcentaje de los habitantes de países en desarrollo está poniendo freno a la expansión del comercio internacional y de la integración financiera mundial dentro del proceso conocido como globalización. Para González (2019) parece que se ha dado comienzo a una nueva era o, al menos, la transición hacia un nuevo escenario en el que los flujos de capitales y el comercio internacional tendrán un papel menos relevante.

González señala que situaciones tales como: el voto a favor del BREXIT, la victoria de Donald Trump, el auge de los partidos populistas que defienden el proteccionismo o el cierre de las fronteras, son una clara muestra de que en las economías avanzadas hay una parte cada vez mayor de la población que no está a gusto con la situación actual y algunos empiezan a hablar de desglobalización.

En ese mismo sentido, Witt (2019) define la desglobalización como el proceso de debilitamiento de la interdependencia entre las naciones. Para muchos, la globalización comienza de manera "oficial" con el nacimiento de la Organización Mundial del Comercio (omc) en 1995, después de más de una década de negociaciones internacionales. La OMC es el organismo multilateral que se encarga de las reglas globales para comercio entre naciones, del que se hacen parte 164 miembros que representan el 98\% del comercio mundial. En noviembre de 1996, la omc, en conjunto con el Banco Mundial y el Fondo Monetario Internacional (FMI), emitieron una declaración para trabajar de manera cooperada por una política económica global coherente, buscando las bases de una prosperidad global (González, 2019).

González afirma que la globalización comercial llegó a su peak entre el año 2007 y 2010, y por su parte, la globalización de las inversiones extranjeras directas lo hizo entre 2007 y 2011. Por ende, el profesor Witt afirma que esta evidencia sugiere que estamos en 
una era de desglobalización, en la que llevamos una década de descenso de la actividad comercial global.

Adicional a su estudio, otros análisis, como el presentado por el medio de comunicación Británico The Economist, argumenta que la globalización se ha detenido o retraído a partir de la crisis financiera del 2008, después de más de dos décadas de constante aumento e integración en los flujos de los mercados globales. Frente a este hecho, se intentaron implementar diferentes medidas, en las que el proteccionismo aparecía como una alternativa posible para la recuperación de las economías del mundo. Sin embargo, irónicamente, la aplicación de este tipo de medidas va en aumento desde el año 2008, tal como lo analiza el Dr. Rumki Majumdar, lo que finalmente resulta en limitaciones al comercio transfronterizo de bienes y servicios (The Economist, 2019).

En diferentes partes del mundo se han venido fortaleciendo iniciativas que claman por la desglobalización. Entre ellas, por ejemplo el movimiento "slow food", creado en 1989 para generar conciencia de la procedencia de los alimentos que se consumen; de cómo se afectan nuestras decisiones de consumo, y además, busca prevenir la desaparición de las tradiciones y culturas gastronómicas locales, y contrarrestar el aumento de la vida rápida. También hay investigaciones como la de Berit Nygard y Oddveig Storstad, en donde se encuentra que los consumidores tienen una preferencia natural por productos locales, al ser percibidos tanto más sanos como más seguros que los productos importados (González, 2019).

De acuerdo a González (2019), el hecho de que estemos en una "era de desglobalización" no significa que es el fin de los negocios internacionales. Pero sí supone mirar la realidad actual con diferentes lentes a la globalización. En su investigación, el profesor Witt concluye que este escenario actual supone tener en consideración estimaciones políticas estratégicas para las empresas multinacionales que afectan la sostenibilidad política de la organización; las dinámicas organizacionales de las cadenas de valor, en cuanto a su especialización, y el papel del contexto nacional; además de las modificaciones necesarias frente a las decisiones sobre estrategias, estructuras y comportamientos en el escenario global. Por ende, las decisiones sobre ubicación de las operaciones, entran nuevamente a tener consideraciones sobre poderes militares y políticos, que influyen en los patrones de inversión o supervivencia en condiciones de des globalización. (Witt, 2019). 


\section{NeOproteccionismo:}

El Proteccionismo Económico es definido como una doctrina económica opuesta al librecambismo, que intenta favorecer la producción nacional frente a la competencia extranjera, haciendo pagar impuestos por la importación de productos extranjeros y favoreciendo a los nacionales con medidas especiales (Boulocq, 2019).

Sin embargo, el nacimiento del concepto de neoproteccionismo está muy relacionado a la forma en la cual el actual gobierno de Estados Unidos viene planteando su política exterior, basada en acciones tales como la construcción de muros o premisas como la de "América First". (Fandiño, 2020).

En su discurso de posesión como Presidente, el 20 de enero del 2017, Trump se refirió de la siguiente manera:

"Durante muchas décadas hemos enriquecido la industria extranjera a expensas de la industria estadounidense [...] y hemos gastado billones y billones de dólares en el extranjero, mientras que la infraestructura de Estados Unidos se ha deteriorado. Hemos hecho ricos a otros países, mientras que la riqueza, la fortaleza y la confianza de nuestro país se han disipado en el horizonte. Una por una, las fábricas cerraron y abandonaron nuestras costas, sin siquiera pensar en los millones y millones de trabajadores estadounidenses que quedaron atrás. La riqueza de nuestra clase media ha sido arrancada de sus hogares y luego redistribuida en todo el mundo. Pero eso es el pasado. Y ahora solo estamos mirando hacia el futuro ("The full text of Donald Trump's inauguration speech", 2017); (Torres, 2019).

De acuerdo a la UnCTAD, la lentitud del crecimiento en la economía mundial junto con el reciente auge del populismo y los sentimientos nacionalistas en países tales como Estados Unidos y el Reino Unido, dieron lugar a medidas proteccionistas, lo que ha tenido grandes repercusiones en el comercio internacional y la integración económica (UNCTAD, 2019).

Tomando como referencia a la potencia mundial del siglo XX, como lo es Estados Unidos, su mandatario, Donald Trump ha venido enfocando su administración en la renegociación del NaFTa, en desarrollar medidas y chantajes contra China, los cuales han ido en aumento hasta llegar a amenazar con extenderlas a todas las importaciones venidas del gigante de Asia. De igual forma, frente al continente europeo, y tras la aprobación de tarifas arancelarias globales a las exportaciones de acero y aluminio, se empezó a evaluar la subida de aranceles a la importación de vehículos.

Otra forma de neoproteccionismo ha sido el pronunciamiento de la Casa Blanca contra los acuerdos 
regionales, como el Acuerdo Transpacífico de Cooperación Económica (тTP, por sus siglas en inglés), donde Estados Unidos ha procedido a renegociar acuerdos comerciales bilaterales, que no fueran beneficiosos para su economía. Siendo evidente, de esta forma, que en tales negociaciones se ha impuesto el dominio y fuerza de su economía frente a cada país con que establece dichos acuerdos.

Para algunos autores, tales como Sagastume (2019), el gobierno de Trump ha renegociado los acuerdos comerciales de carácter bilateral para negociar e instaurar convenios propicios para aquellos bienes y servicios que consideren que son relevantes para promover la producción en su país, con el objetivo de generar efectos multiplicadores internos a favor del empleo, la reducción de su déficit de comercio exterior y volver a ser la gran potencia.

De igual forma, decidió declarar una "guerra comercial" contra China, buscando -a través del proteccionismo- mantener la superioridad tecnológica de su país. La voluntad política del Presidente Trump y su equipo muestra estar mucho más interesada en protegerse comercialmente y organizar los asuntos internos de su país, que en mantener un liderazgo internacional basado en la promoción de libertades e innovación tecnológica (Van Ham, 2018), en la medida que considera que la fortaleza interna es la que confiere fortaleza externa, y no al revés. Paradójicamente, las defensas políticas de las virtudes del libre comercio están quedando en manos de China (Torres, 2019).

\section{Desregionalización:}

Un documento publicado por el Departamento de Sociología de la Universidad de Nueva York, define la desregionalización como "el proceso de disminución de la interdependencia y la integración entre ciertas unidades en todo el mundo, por lo general en los Estados-Nación" (New York University Department of Sociology, 2019. P. 14).

Este documento señala que este término ha venido siendo utilizado para representar los ciclos de la historia mercantil, cuando el comercio y la inversión entre países se reducen. Ello en contraste con la globalización, en que las unidades acrecientan su integración cada vez más y, por lo general, extiende el tiempo entre períodos de globalización (New York University Department of Sociology, 2019).

El caso más sobresaliente de desregionalización es el presentado en el Reino Unido luego del inesperado triunfo del BREXIT en el referéndum convocado por el gobierno de David Cameron en el Reino Unido, en junio de 2016, lo cual ha generado un 
terremoto político y económico sin precedentes en el seno de la Unión Europea, y ha llevado a que varios autores empiecen a hablar sobre un fenómeno denominado desregionalización. En este proceso de desregionalización, el BREXIT ha conducido a la mayor reducción en la integración económica entre los países después de la II Guerra Mundial (Gaviro, 2017).

Otra señal que indica que el mundo entra en un proceso de desregionalización está relacionada con las principales decisiones económicas tomadas por Estados Unidos en los últimos años, contra toda la filosofía aperturista de los tratados de libre comercio que anteriores gobiernos habían negociado, destacándose la renuncia a los más significativos tratados en proceso de implementación: el Transpacífic Partnership, recién acordado por la administración Obama con Japón, Australia, México, Chile, Canadá y otros seis países (excluyendo significativamente a China), mientras también congeló las negociaciones en curso con la Unión Europea para el Transatlantic Trade and Investment Partnership, que sería uno de los mayores tratados de libre comercio vigentes en el mundo (Torres, 2019).

De acuerdo a Torres, a pesar de la importancia de estos dos tratados, que trazaban una ruta estratégica comandada por Estados Unidos, y que deberían ser la referencia internacional obligada para las inversiones y el comercio del siglo xxI, el Presidente Trump optó por desconocerlos y volcar su agenda de negociación económica, primero, hacia algo más cercano: el North American Free Trade Agreement (NAFTA), en cuya renegociación con sus dos socios regionales, Canadá y México, aspira lograr éxitos económicos más inmediatos y más tangibles (Torres, 2019).

\section{Conclusiones:}

El multilateralismo es un concepto largamente divulgado dentro de las Relaciones Internacionales, ya que refiere a la situación de varios Estados-Nación trabajando conjuntamente en los mismos aspectos. Dirigentes políticos y gobernantes nacionales destacan que gracias a este instrumento se logran acuerdos mediante el diálogo, en temas de la agenda internacional como cambio climático, seguridad y paz, desarrollo y los Objetivos de Desarrollo Sostenible.

Lamentablemente, en los últimos años y en víspera del inicio de la segunda década del siglo xxi no se ha visto un verdadero multilateralismo, sino un unilateralismo disfrazado de multiplural. Varios Estados-Nación considerados potencias han hecho gravitar las cuestiones de interés internacional en base a sus intereses nacionales particulares y mediante el proceso de 
coaliciones; un ejemplo de ello es Estados Unidos y el Reino Unido.

El argumento central de este documento fue que las relaciones internacionales han sufrido una transformación en los últimos años, generada por una crisis del multilateralismo heredado del período de la post guerra. Esta crisis del multilateralismo se ha visto materializada mediante un proceso de des globalización de la economía mundial, fundamentada principalmente en políticas neoproteccionistas que han conducido a una desregionalización.

En los últimos años, la economía mundial ha entrado en un período de desglobalización, es decir, de retroceso en los flujos internacionales de mercancías, servicios, capitales y personas. La desglobalización de estos últimos años ha tenido varias causas principales, donde destaca la desaceleración económica, que afecta de forma directa y negativa al comercio, las inversiones y los movimientos de capital; el aumento del proteccionismo y, en general, la ofensiva contra el multilateralismo, cuya manifestación más importante es la nueva política de Estados Unidos; la regresión en las cadenas globales de valor. Estas habían experimentado un crecimiento constante desde principios de los años ' 90 , llegando a suponer en torno a un 50\% del comercio mundial, pero en estos últimos años han comenzado a perder fuerza. Diversos factores explican el retroceso en las cadenas globales de valor. Por un lado está el proteccionismo. Por otro, el aumento de los salarios y del nivel de renta en los países en desarrollo que eran destinos de deslocalizaciones.

El mundo de la segunda década siglo xxi será demasiado interdependiente y exigirá un diferente marco de colaboración que, además de los necesarios equilibrios de poder, tenga en cuenta la multiplicidad de los desafíos actuales y la necesidad de reafirmar un modelo normativo sobre una base más democrática.

\section{Bibliografía}

Baldwin, R. (2016). The Great Convergence. Harvard University Press.

Barkin, J.S. \& Cronin, B. (1994). The state and the nation: changing norms and the rules of sovereignty in international relations. International organization, 48(1), 107-130.
Brunnée,J. (2018). Multilateralism in Crisis. Proceedings of the ASIL Annual Meeting,112,335-339. doi:10.1017/amp.2019.35 Boulocq, P. (2019). Neoproteccionismo encubierto en la órbita de la OMC: EE.UU. y las medidas que afectaron a las importaciones de limones frescos de origen argentino (2001-2018). Master's thesis. 
Buenos Aires: FLACSO-Sede Académica Argentina.

Coppelli Ortiz, G. (2018). La globalización económica del siglo XXI. Entre la mundialización y la desglobalización. Estudios Internacionales, 50(191), 57-80.https:// dx.doi.org/10.5354/0719-3769.2019.52048

Dabat Latrubesse, A., y Leal Villegas, P. (2019). "Ascenso y declive de Estados Unidos en la hegemonía mundial”. Problemas del Desarrollo. Revista Latinoamericana de Economía, Vol.50. №199.

Eilstrup-Sangiovanni, M., \& Hofmann, S.C. (2019). Of the contemporary global order, crisis, and change. Journal of European Public Policy, 1-13.

Fandiño Sánchez, A.E. (2020). El neoproteccionismo, ¿una nueva tendencia mundial?. Bachelor's thesis. Fundación Universidad de América.

Gaviro, J.M. (2017). Brexit: Efectos económicos en un escenario incierto. La Albolafia: Revista de Humanidades y Cultura (12), 55-74.

García Moritan, M. (2019). La Crisis del Multilateralismo. El Telégrafo.

Guillén, A. (2018). El gobierno de Trump frente a la crisis global y el estancamiento económico. Cuadernos de Economía Crítica, 4(8), 101-127.

González, M. (2019) La Era de la desglobalización.Dinero. Recuperado de: https:// www.dinero.com/opinion/columnistas/articulo/la-era-de-la-desglobalizacion-por-maria-alejandra-gonzalez-perez/272967

Hernández, P. y Jaramillo, Y. (2017). Tendencias de investigación en negocios internacionales (2012-2016). Cuadernos
Latinoamericanos de Administración, vol. 13, no 25, p. 9-18.

Hidalgo Gallo, R. (2018). Tendencias del comercio internacional de bienes, políticas comerciales y negociaciones multilaterales. Economía y Desarrollo, 159(1), 135-153.

Ikenberry, G.J. (2018). The end of liberal international order? International Affairs, 94(1), 7-23.

New York University Department of Sociology (2013). The Sociology Project. United States of América: Pearson. p.569. ISBN 978-0-205-09382-3.

Newman, E., y Stefan, C.G. (2019). Normative Power Europe? The EU's Embrace of the Responsibility to Protect in a Transitional International Order.JCMS: Journal of Common Market Studies.

Polanco Sagastume, M.L. (2019). ¿Existe un proceso de des globalización económica?. IIES. Facultad de Ciencias Económicas, Universidad de San Carlos de Guatemala.

Rüland,J. (2020). "Multilateralism Light"?. The Asia-Europe Meeting (ASEM) and the Rescue of a Rules-Based International Order.

Serbin, A. (2018). América Latina y el Caribe frente a un nuevo orden mundial: crisis de la globalización, reconfiguración global del poder y respuestas regionales. América Latina y el Caribe, 13.

Steinberg, F. (2018). Gobernanza de la globalización. Nueva Revista, № 162.

Torres, J. (2019). Declive del liderazgo de EE. UU. con Trump y ascenso de China como opción del multilateralismo global. Razón Crítica, (7), 139-175. 
Van Ham, C. y Seim, B. (2018). Strong states, weak elections? How state capacity in authoritarian regimes conditions the democratizing power of elections. International Political Science Review, 39(1), 49-66.

Witt, M.A. (2019). De-globalization: Theories, predictions, and opportunities for international business research.Journal of International Business Studies, 1-25.

Zelicovich, J. (2019). La crisis de la OMC y el trilema de las negociaciones comerciales internacionales. Relaciones Internacionales, 92(1), 1-21. 